should have been performed in patients prior to dronedarone treatment (GROUP A), those who started the treatment before the alert (group B), and finally patients who discontinued this semester (GROUP C). In group B patients we checked whether the ongoing controls specified in the alert had been done. Similarly, in Group A patients we checked whether the start of treatment controls had been done (renal and hepatic function before and the week of the start of treatment). Serum creatinine concentration was considered valid as a cheque of renal function; transaminase levels were suitable for the liver function test. Selene (clinical history management software) and Agora Plus (primary integrated medical recordhospitalisation management software) were used to retrieve the serum concentrations.

Results We examined 72 clinical histories. Group A contained 17 patients. Only $5 \%$ had liver and kidney function tests as required by the Competent Authority. In group B (48 patients), 31.2\% had none of the controls required. Only $6.2 \%$ of patients had a creatinine cheque. Only $4.1 \%$ of patients had a liver function cheque. In Group C with 7 patients (two deaths), $71.4 \%$ had no analytical controls of any kind, and in only $14.2 \%$ were renal function tests performed.

Conclusions The degree of compliance with tests required by the Health Authority in patients taking dronedarone is very low. It seems necessary to review and improve the system of drug alerts to physicians, and the pharmaceutical care of patients seen in primary healthcare. Computer systems such as the Agora Plus that integrate primary and hospitalisation data are critical for this type of monitoring.

No conflict of interest.

\section{GRP-119 MOST FREQUENT DRUG-RELATED EVENTS DETECTED BY PHARMACEUTICAL ANALYSIS OF COMPUTERIZED PHYSICIAN ORDER ENTRY AND PROPOSED SOLUTIONS}

doi:10.1136/ejhpharm-2013-000276.119

N Chu, T Gorce, V Duhalde, P Cestac, M Vie, B Bellon. Toulouse University Hospital, Haute Garonne, Toulouse, France

Background In 2012, Toulouse University hospital implemented a Computerized Physician Order Entry (CPOE) system in two digestive surgery departments (41 inpatient beds). Clinical pharmacists in the wards contribute to safeguarding the medication process by reviewing prescriptions.

Purpose To highlight recurrent and avoidable drug-related problems identified by pharmaceutical analysis of CPOE and to raise physicians' awareness regarding these prescription problems.

Materials and Methods From April to July 2012, Pharmaceutical Interventions (PIs) concerning prescription problems were recorded in the CPOE according to the codification defined by the working group of the French Society of Clinical Pharmacy. We extracted the following data from the CPOE: drugs, type of problems and PIs. We identified the main prescription problems and drugs involved. After data analysis, preventive measures were submitted to the physicians

Results 2396 prescriptions were analysed and 450 Pharmaceutical Interventions (PIs) were accepted by physicians (18.8\%). Main prescription problems concerned analgesics (52 PIs made): inappropriate administration and dosage errors; heparins (31 PIs): dosage errors; antiemetics (24 PIs): dosage errors and drug-drug interactions; antibiotics (16 PI): inappropriate prescription. To prevent these problems, a multi-disciplinary group was set up with physicians, nurses and pharmacists. This group has reviewed standardised order sets and has developed a pocket guide to help new residents while prescribing.
Conclusions This study describes the most frequent CPOE problems. Communication and collaboration with physicians and nurses are the key to reducing avoidable adverse drug events and to safeguarding CPOE.

No conflict of interest.

\section{GRP-120 MULTIDISCIPLINARY INTERVENTION: INTRATHECAL AND INTRAVENTRICULAR CHEMOTHERAPY IN PAEDIATRICS}

doi:10.1136/ejhpharm-2013-000276.120

'L Guerrero-Aznar, ' 'A Casado-Jimenez, ${ }^{2} \mathrm{R}$ Cumplido-Corbacho, ${ }^{2} \mathrm{~S}$ Romero-Carmona 'A Villalba-Moreno, 'L Fernandez-Fernandez. 'Hospital Universitario Virgen del Rocío, Pharmacy, Seville, Spain; ${ }^{2}$ Hospital Universitario Virgen del Rocío, Pediatrics, Seville, Spain

Background A communications system was designed after notification of two errors in two months, in intrathecal and intraventricular chemotherapy in paediatric oncohaematology: prescribing by protocol, consultation sheet standardised and computerised; transcription using the Farmis integrated system for chemotherapy and preparation centralised in the pharmacy after standardisation, and administration with a double cheque. Functions were established and detailed in each process to all groups involved.

Purpose To conduct a retrospective observational descriptive study to cheque compliance with the intrathecal and intraventricular rules at each level: prescription, transcription, preparation, distribution and administration and to analyse any change in the errors made with intrathecal and intraventricular chemotherapy before and after the new system was implemented.

Materials and Methods Each of the processes in the system was tracked, during the year after the intervention - July 2011. Prescriptions were analysed through electronic medical records, Farmis, nursing and pharmacy records.

Medicines error reporting to the Safety Commission was monitored during the year after the implementation. The error rate was determined by comparing the two months prior to the intervention.

Results 167 prescriptions were checked, 133 intrathecals and 34 intraventriculars. The professionals involved were monitored $100 \%$ in all processes, except the administration checklist by neurosurgeons, which was only $62.5 \%$ checked. The error rate reported by number of prescriptions went from 0.14 in the previous two months to 0.006 in the year after intervention.

Conclusions There has been high system monitoring by all professionals involved. The number of medicines errors became lower in the post-intervention period. Thus, centralization and standardisation of intrathecal and intraventricular chemotherapy has helped increase patient safety.

No conflict of interest.

\section{GRP-121 MULTIDISCIPLINARY MONITORING OF PSYCHIATRIC MORBIDITY OF HCV-INFECTED PATIENTS TREATED WITH INTERFERON AND RIBAVIRIN}

doi:10.1136/ejhpharm-2013-000276.121

'M Cabré, 'N Rudi, ${ }^{2} \mathrm{C}$ Pontes, ${ }^{3} \mathrm{M}$ Vergara, 4 I Parra, ${ }^{1} \mathrm{M} 0$ Gorgas. 'Corporación Sanitaria Parc Tauli, Pharmacy, Sabadell, Spain; ${ }^{2}$ Corporación Sanitaria Parc Tauli, Clinic Pharmacology, Sabadell, Spain, ${ }^{3}$ Corporación Sanitaria Parc Tauli, Digestive Diseases, Sabadell, Spain; ${ }^{4}$ Corporación Sanitaria Parc Tauli, Mental IIIness, Sabadell, Spain

Background Treatment of hepatitis $\mathrm{C}$ virus (HCV) infection with pegylated interferon and ribavirin may induce psychiatric disorders, which may result in poor adherence and response to antiviral treatment. 
Purpose We aimed to describe the incidence of neuropsychiatric disorders in a cohort of HCV-infected patients treated with interferon and ribavirin, and their impact on treatment adherence and viral response rate (SVR).

Materials and Methods Data from a cohort of HCV patients who visited an outpatient pharmacy service (OPS) included all adult patients mono-infected with HCV who had completed treatment in 2010. Monitoring of neuropsychiatric disorders was assessed at weeks $0,4,12,24,48$, and 72 through the selfadministered questionnaires Hospital Anxiety and Depression Scale (HADS) and General Health Questionnaire (Goldberg). Adherence to treatment was assessed by counting drugs dispensed and patient reporting. Virological response was determined by the physician according to standard criteria.

Results Of the 76 patients included, 19 (25\%) had a pre-existing psychiatric disorder, mostly depression and anxiety. The incidence of medically-confirmed neuropsychiatric disorders was $33 \%$ $(\mathrm{n}=25)$, with a peak of abnormal results in the tests in week 12 . Patients with and without pathological scores did not differ in baseline characteristics, except for pre-existing psychiatric disorder $[60.0 \%$ vs. $7.8 \%$, respectively ( $p<0.001)$. Antidepressants and/or anxiolytics were prescribed to $48 \%$ of patients with medically confirmed disorders $(n=12)$. Overall, $43 \%$ of patients achieved an SVR. Strict adherence ( $96 \%$ vs. $90 \%$; p $=$ NS) and SVR (39\% vs. $52 \%$; $\mathrm{p}=\mathrm{NS}$ ) were similar in patients with or without medically confirmed disorders.

Conclusions Patients often develop neuropsychiatric disorders during interferon therapy. Neuropsychiatric side effects had a nonsignificant effect on adherence to treatment and attainment of SVR. Multidisciplinary monitoring provided during the treatment of hepatitis $\mathrm{C}$ can contribute to early detection and management of neuropsychiatric disorders and to improve integrated patient care.

No conflict of interest.

\section{GRP-122 NEW PERSPECTIVES OF HOSPITAL PHARMACISTS' ROLE IN COMMUNICATING PHARMACEUTICAL POLICY TO HEALTHCARE PROFESSIONALS AND PATIENTS IN GREECE}

doi:10.1136/ejhpharm-2013-000276.122

'K Nikou, ${ }^{2} \mathrm{~K}$ Perdikouri. 'General Hospital of Chest Diseases "SOTIRIA", Pharmacy, Athens, Greece; '2Spiliopoulio Hospital, Pharmacy, Athens, Greece

Background Given the burden of unfortunate economic conditions in Greece, hospital pharmacists find themselves at the crossroads of different approaches to patient treatment options, trying to ensure the patient gets the best treatment from both the financial and medical point of view.

Purpose To highlight the importance of hospital pharmacists' role in the 'communication chain' within and beyond the hospital.

Materials and Methods Everyday practise experience (e.g. frequently asked questions) at the hospital including communication with patients, doctors and the hospital administration has been taken into account in addition to the Hellas Health IV survey (sample size: 1008 persons).

Results Everyday experience shows that the majority of patients don't really understand why there is a change (e.g. generic substitution) in their treatment and they insist on it not being changed. The survey showed that $63 \%$ didn't know the meaning of generic medicine and only $26 \%$ of those who knew were sufficiently informed to realise that the generic version is therapeutically equivalent to the original brand.

Conclusions It is important for hospital pharmacists to be aware of developments in health care communication, for example to be able to recommend on-line resources to patients about the treatment of their disease and use of generic drugs. Moreover they have to develop and improve the required skills in medical and financial issues as they are important stakeholders in the chain of health promotion in the hospital.

No conflict of interest.

\section{GRP-123 NON FORMULARY DRUG MANAGEMENT - ABSURD OR REASONABLE?}

doi:10.1136/ejhpharm-2013-000276.123

A Kellermann, M Trojan, A Müller, C Querbach, R Bernard. Klinikum rechts der Isar der TUM München, Pharmacy, München, Germany

Background Non-formulary drugs are prone to cause medication errors due to their less common use in the daily routine on the ward. Therefore non-formulary drug (NFD) management in the hospital pharmacy includes checking the dose and indication which is usually very time consuming. In 2010 the drug information centre had to deal with 12,903 prescriptions for NFDs.

Purpose Loss of relevant drug information at the interface between pharmacy and ward has been observed in some cases. Therefore a survey was performed to detect information gaps. Did the pharmacist's recommendation reach the medical staff?

Materials and Methods During a period of four weeks all NFD prescriptions were documented concerning the type of medicine. If a treatment-relevant intervention (e.g. dose correction) was made the trainee pharmacist visited the ward to clarify if the pharmacist's advice was received. In addition the medical staff were interviewed about the general procedure of information transfer within the ward staff.

Results 1158 NFDs were ordered. Out of these 261 required extensive action with pharmacist intervention. 256 interventions were accepted on the ward and only 5 were rejected. In only one case out of these the pharmacist's information had to be resupplied to the ward as it had not reached the staff. The survey showed a very high acceptance $(98.1 \%)$ of the drug information provided. 83 drugs within the ATC Code "antibiotics for systemic use" were particularly counselling-intense. Dosing problems were the most frequent drug-related problem (52). Information transfer within the ward turned out to be highly inhomogeneous.

Conclusions The pharmaceutical advice offered to the ward was accepted to a very high percentage. To prevent information loss on the ward a standardised system for information transfer amongst the staff needs to be established.

No conflict of interest.

\section{GRP-124 NON-BIOLOGICAL COMPLEX DRUGS AND THEIR REGULATORY APPROACH - OF CONCERN FOR HOSPITAL PHARMACISTS AND MEDICINES MANAGEMENT?}

doi:10.1136/ejhpharm-2013-000276.124

${ }^{1} \mathrm{G}$ Borchard, ${ }^{2} \mathrm{JB}$ Rottembourg, ${ }^{3 B} \mathrm{~B}$ Flühmann, ${ }^{4} \mathrm{~S}$ Mühlebach. 'Uni Geneva, School of Pharmacy Geneva-Lausanne, Geneva, Switzerland; ${ }^{2}$ Hospitalier Pitié-Salpétrière, Nephrology, Paris, France; ${ }^{3}$ Vifor Fresenius Medical Care Renal Pharma Ltd, Anemia TA, St. Gallen, Switzerland; ' $V i f o r$ Pharma Ltd, Global Regulatory Affairs, Glattbrugg, Switzerland

Background Intended copies of originator medicinal products (MPs) are categorised as generics or biosimilars (complex large molecular biological MPs) with distinct regulatory pathways for marketing authorization. In recent years, a new category of non-proteins and non-biotech-derived MPs has emerged, the non-biological complex drugs (NBCDs) comprising IV iron 